\title{
Pneumococcal conjugate vaccine
}

\section{A newer vaccine available in India}

Ramesh Verma* and Pardeep Khanna

Department of Community Medicine; Pt. B.D. Sharma PGIMS; Rohtak, Haryana India
Keywords: Streptococcus pneumonia, pneumonia, otitis media, mortality, vaccines

Submitted: 04/30/12

Accepted: 05/05/12

http://dx.doi.org/10.4161/hv.20654

*Correspondence to: Ramesh Verma;

Email: dr.rameshverma@yahoo.co.in
Streptococcus pneumoniae, or "pneu$\checkmark$ mococcus," causes pneumonia and infections of the brain and blood that are responsible for significant mortality in children under five years as well as in the elderly. Pneumococcal diseases are a major public health problem worldwide. S. pneumoniae is responsible for $15-50 \%$ of all episodes of community-acquired pneumonia, 30-50\% of all cases of acute otitis media, and a significant proportion of bacterial meningitis and bacteremia. S. pneumoniae kills at least one million children under the age of five every year, which is more than malaria, AIDS and measles combined. More than $70 \%$ of the deaths are in developing countries. In 2007, pneumococcal pneumonia was the leading infectious killer of children worldwide. Perhaps more importantly, pneumonia remains the leading killer of children in India. A recent UNICEF publication estimated that 410,000 children under age five years die of pneumonia each year in India, and recent data shows that an estimated $25 \%$ of all child deaths in India are due to pneumonia. The fact that this high burden of pneumonia has remained undiminished in India in spite of economic growth and decline in child mortality due to other diseases is a reminder of the importance of tackling pneumonia head-on with dedicated resources. The burden of pneumococcal meningitis, which constitutes about half of all childhood meningitis cases in most settings and a greater proportion of meningitis deaths, makes it difficult to avoid the conclusion that the pneumococcus is responsible for one million child deaths each year. Global
Alliance for Vaccine and Immunization (GAVI) has offered to supply PCV at a cost of $0.15-0.30 \mathrm{USD} /$ dose to India for inclusion in the national immunization schedule and commits to extending this support until the year 2015. Pneumococcal vaccination in not recommended in children aged five and above.

\section{Pneumococcal Conjugate Vaccine: A Newer Vaccine Available in India}

Streptococcus pneumoniae or "pneumococcus," causes pneumonia and infections of the brain and blood that is responsible for mortality in children under five years. Pneumococcal diseases are a major public health problem worldwide. ${ }^{1} S$. pneumoniae is a Gram-positive encapsulated cocci. Based on differences in the composition of the polysaccharide capsule, about 90 serotypes have been identified. The capsule is an essential virulence factor. The majority of cases of pneumococcal diseases in infants is associated with a small number of these serotypes, which vary by region. Pneumococci are transmitted by direct contact with respiratory secretions from patients and healthy carriers. Although transient nasopharyngeal colonization rather than disease is the normal outcome of exposure to pneumococci, bacterial spread to the sinuses or the middle ear, or bacteremia following penetration of the mucosal layer, may occur in persons susceptible to the involved serotype. Pneumococcal resistance to essential antimicrobials such as penicillins, cephalosporins and macrolides is a 
serious and rapidly increasing problem worldwide. Facilities for laboratory diagnosis of $S$. pneumoniae, based on growth in traditional culture media, are available in laboratories for routine clinical microbiology, whereas serotyping is performed only in reference laboratories. ${ }^{1}$

S. pneumoniae is responsible for $15-50 \%$ of all episodes of communityacquired pneumonia, 30-50\% of all cases of acute otitis media and a significant proportion of bacterial meningitis and bacteremia. ${ }^{2-4}$ However, based on available data, acute respiratory infections kill an estimated 2.6 million children less than five years of age annually. $S$. pneumoniae kills at least one million children under the age of five every year, a sum that is more than malaria, AIDS and measles combined. More than $70 \%$ of the deaths are in developing countries. 5 In Europe and the US, pneumococcal pneumonia is the most common community-acquired bacterial pneumonia, estimated to affect approximately 100 per 100,000 adults each year. The corresponding figures for febrile bacteremia and meningitis are 15-19 per 100000 and 1-2 per 100,000, respectively. ${ }^{1}$ The risk for one or more of these disease manifestations is much higher in infants and elderly people. Even in economically developed regions, invasive pneumococcal disease carries a high rate of mortality; for adults with pneumococcal pneumonia the mortality rate averages $10-20 \%$, while it may exceed $50 \%$ in the high-risk groups. Pneumonia is by far the most common cause of pneumococcal death worldwide.

Out of 90 serotypes of $S$. pneumoniae, a handful is responsible for most cases of invasive pneumococcal disease (IPD). Serotypes 1, 2, 3, 4, 5, 6A, 6B, 7F, 8, 9A, $9 \mathrm{~N}, 9 \mathrm{~V}, 10 \mathrm{~A}, 12 \mathrm{~F}, 14,15 \mathrm{~B}, 18 \mathrm{C}, 19 \mathrm{~A}$, $19 \mathrm{~F}$ and $23 \mathrm{~F}$ are responsible for $85 \%$ of invasive pneumococcal disease in the developed world. ${ }^{4}$ Children under the age of two years are at greatest risk for invasive pneumococcal disease. Results of the Invasive Bacterial Infection Surveillance (IBIS) study in patients with invasive pneumococcal disease (IPD) indicate that serotypes $1,2,3,4,5,6 \mathrm{~A}, 6 \mathrm{~B}, 7 \mathrm{~F}, 8$, 9A, 9N, 9V, 10A, 12F, 14, 15B, 18C, 19A, $19 \mathrm{~F}$ and $23 \mathrm{~F}$ are the most prevalent, with serotypes 1 and 5 accounting for $30 \%$ of invasive pneumococcal disease. ${ }^{6}$ It is also known that the serotypes causing pneumonia and otitis media differ from those causing invasive pneumococcal disease and usually reflect those serotypes present in nasopharyngeal carriage.

Studies conducted in many parts of the developing world have consistently shown the two leading causes of bacterial pneumonia to be $S$. pneumoniae (pneumococcus) and Hemophilus influenzae type $b^{7,8}$ In 2007, pneumococcal pneumonia was the leading infectious killer of children worldwide. Perhaps more importantly, pneumonia remains the leading killer of children in India. A recent UNICEF publication estimated that 410,000 children under age five years die of pneumonia each year in India, and a recent data shows that an estimated $25 \%$ of all child deaths in India are due to pneumonia.?

The fact that this high burden of pneumonia has remained undiminished in India in spite of economic growth and decline in child mortality due to other diseases is a reminder of the importance of tackling pneumonia head on with dedicated resources. The burden of pneumococcal meningitis, which constitutes about half of all childhood meningitis cases in most setting and a greater proportion of meningitis deaths, makes it difficult to avoid the conclusion that the pneumococcus is responsible for one million child deaths each year. ${ }^{10}$ This is more than any other pathogen, including Plasmodium falciparum. Clearly this is the most important new target for childhood vaccination in developing countries. As advocates for child health, individual pediatricians and the Indian Academy of Pediatrics should urge the Indian Government to develop a process and timeline for introducing pediatric pneumococcal vaccine. Meta-analysis of four high-quality, randomized, controlled trials of pneumococcal conjugate vaccines suggests that about $30-40 \%$ of all severe pneumonia in children are likely to be pneumococcal in origin. ${ }^{11}$ Based on the available data and policy options before it, India can take several important steps to prevent pneumonia and improve child survival through the expanded use of life-saving pneumonia vaccines.

\section{Pneumococcal Conjugate Vaccines (PCVs)}

PCVs were developed primarily to address the problem of low immunogenicity of the polysaccharide vaccine in children below the age of two who are at high risk for pneumococcal disease. Conjugation of the pneumococcal polysaccharide of varying number of serotypes has been done with CRM197 protein, protein D of non-capsulated Hib, DT and TT and finally Meningococcal Outer Member Protein (OMP). The only PCV licensed to date in India is CRM197 PnC-7v (henceforth referred to as PCV7, Pfizer) containing polysaccharide antigen of serotypes 4, 6B, 9V, 14, 18C, $19 \mathrm{~F}$ and $23 \mathrm{~F}$ linked to CRM197.12 The landmark randomized controlled northern California Kaiser Permanente Vaccine (NCKP) trial in the US in around 40,000 children with three primary doses at 2, 4 and 6 months and a booster at 12-15 months demonstrated $95 \%$ protection against IPD due to vaccine serotypes, $89 \%$ protection against any serotype, $4 \%$ protection against clinically diagnosed pneumonia, 30\% protection against radiological pneumonia, $6 \%$ protection against acute otitis media and $20 \%$ protection against placement of tympanostomy tubes over a five-year follow-up period. No serious adverse effects were noted, while only $15-20 \%$ of the vaccinees had local side effects. ${ }^{13}$

Till recently only 7-valent pneumococcal conjugate vaccine (PCV7) and unconjugated pneumococcal polysaccharide vaccine (PPSV 23) were available in India. However, in mid 2010, a new 13-valent conjugate vaccine (PCV13) was launched in India. PCV 13 has replaced PCV 7 world over, including India. PCV 13 contains six additional strains (i.e., 1, 3, 5, 6A, 19A and 7F), which protect against the majority of the remaining pneumococcal infections. Another PCV, a 10 -valent pneumococcal conjugate vaccine (PCV10) from GSK is now available internationally and soon will be available in India too. ${ }^{14}$ 


\section{Recommendations for Pneumococcal Conjugate Vaccines in India}

The burden of pneumococcal disease is the greatest among the underprivileged children in India. The PCVs are thus of public health importance and ideally should be available to all children. However the high cost of PCVs and the limited coverage of the currently available vaccine are impediments. GAVI has offered to supply PCV at a cost of $0.15-0.3 \mathrm{USD} /$ dose to India for inclusion in the national immunization schedule and commits to extending this support until the year 2015. ${ }^{11}$ The risk of invasive pneumococcal disease is significantly lower in healthy children above the age of two years and thus benefit achieved with vaccination of these children is likely to be lower. Vaccination with single dose of PCV vaccine may be considered in children aged $2-5$ years. ${ }^{15}$ Pneumococcal vaccination in not recommended in children aged five and above. The recommended dosage for PCV 13 is $0.5 \mathrm{~mL}$, and the vaccination route is intramuscular.

\section{Schedule for Pneumococcal Conjugate Vaccines ${ }^{16}$}

Infants and children who have not previously received PCV7 or PCV13. Indian Academy of Pediatrics recommends three doses at 6,10 and 14 weeks with a booster at 15 months. Infants receiving their first dose at age $<11$ months should receive three doses of PCV13 at intervals of approximately four weeks with a booster at 15 months. Children aged 12-23 months should receive two doses with an interval of at least eight weeks between doses. Unvaccinated healthy children aged
24-59 months should receive a single dose of PCV13.

Children incompletely vaccinated with PCV7 or PCV13. Infants less than 24 months should receive one or more doses based on the number of doses of PCV7 received to date and the age of the child.

Children who have received four doses of PCV7. A single dose of PCV13 is recommended for all children 14 to 59 months of age.

Children 6 to 18 years of age with high-risk conditions. A single dose of PCV13 may be administered for who are at increased risk for invasive pneumococcal disease because of sickle cell disease, HIV infection or other immunodeficiency state, regardless of whether they have previously received PCV7 or pneumococcal polysaccharide vaccine (PPSV) 23. 


\section{References}

1. Immunization, vaccine and biological. pneumococcal Vaccine World Health Organization (WHO) Available From: www.who.int/vaccines/

2. UNICEF/WHO. Pneumonia: The forgotten killer of children. Geneva: 2006.

3. Dowell SF, Butler JC, Giebink GS, Jacobs MR, Jernigan D, Musher DM, et al. Acute otitis media: management and surveillance in an era of pneumococcal resistance-a report from the Drug-resistant Streptococcus pneumoniae Therapeutic Working Group. Pediatr Infect Dis J 1999; 18:1-9; PMID:9951971; http://dx.doi. org/10.1097/00006454-199901000-00002.

4. Advisory Committee on Immunization Practices. Preventing pneumococcal disease among infants and young children. Recommendations of the Advisory Committee on Immunization Practices (ACIP). MMWR Recomm Rep 2000; 49:1-35; PMID:11055835.

5. Pneumococcal vaccine rolls out in developing world. Vaccine is first to be launched in rich and poor countries simultaneously. Published online 11 February 2011. Nature. Available from: http://www.nature. com/news/2011/110211/full/news.2011.89.html; http://dx.doi.org/10.1038/news.2011.89.

6. Prospective multicentre hospital surveillance of Streptococcus pneumoniae disease in India. Invasive Bacterial Infection Surveillance (IBIS) Group, International Clinical Epidemiology Network (INCLEN). Lancet 1999; 353:1216-21; PMID:10217081.
7. Adegbola RA, Falade AG, Sam BE, Aidoo M, Baldeh I, Hazlett D, et al. The etiology of pneumonia in malnourished and well-nourished Gambian children. Pediatr Infect Dis J 1994; 13:975-82; PMID:7845751; http://dx.doi.org/10.1097/00006454-19941100000008.

8. Shann F. Etiology of severe pneumonia in children in developing countries. Pediatr Infect Dis 1986; 5:247-52; PMID:3952013; http://dx.doi. org/10.1097/00006454-198603000-00017.

9. Thacker N. Integrated management of neonatal and childhood illnesses: a new hope for child survival. Indian Pediatr 2007; 44:169-71; PMID:17413191.

10. Murray CJL, Lopez AD. Global Health Statistics. Boston, Harvard University Press 1996; 288.

11. Levine OS, Cherian T. Pneumococcal vaccination for Indian children. Indian Pediatr 2007; 44:491-6; PMID: 17684301.

12. Vaccine schedule. Provides vaccine alert on you mobile, pneumococcal vaccines 2012. Available from: http://www.vaccineschedule.in/pneumococcal.aspx

13. Eskola J, Kilpi T, Palmu A, Jokinen J, Haapakoski J, Herva E, et al.; Finnish Otitis Media Study Group. Efficacy of a pneumococcal conjugate vaccine agains acute otitis media. N Engl J Med 2001; 344:403 9; PMID:11172176; http://dx.doi.org/10.1056/ NEJM200102083440602.
14. Paediatric on call. Child Health Care. Conjugate pneumococcal vaccine 2011. Available From: http:// www.pediatriconcall.com/fordoctor/diseasesandcondition/immunization_vaccination/pneumococcal_vaccine.asp.

15. Sinhgal T; Indian Academy of Pediatrics Committee on Immunization (IAPCOI). Consensus recommendations on immunization 2008. Indian Pediatr 2008, 45:635-48; PMID:18723905.

16. Yewale V, Choudhury P, Thacker N. IAP Guide Book on Immunization. IAP Committee on Immunization 2009-2011. Available from: http://www.indg. in/health/child-health/IA P \% 20GUIDE \% 20 BOOK\%20ON\%20IMMUNIZATION\%2020092011.pdf. 